\title{
A Prospective Study on the Efficacy of Cognitive Targeted Transrectal Ultrasound Prostate Biopsy in Diagnosing Clinically Significant Prostate
}

\section{Cancer}

Mathisekaran Thangarasu (iD

Sanjay Prakash Jayaprakash (ID

Nivash Selvaraj

Sandeep Bafna (D)

Rajesh Paul

Chandranathan Mahesh

Nitesh Jain

Arunkumar Balakrishnan

Ananthakrishnan Sivaraman

Department of Urology, Apollo Main Hospital, Chennai, Tamil Nadu, India
Correspondence: Mathisekaran Thangarasu Department of Urology, Apollo Main Hospital, 2I Gream's Lane, off Gream's Road, Chennai, 600006, Tamil Nadu, India Email mathisekaran@gmail.com
Purpose: We present our study, done to identify the diagnostic yield of cognitive targeted biopsy using mpMRI data, to diagnose clinically significant prostate cancers, in a cohort of biopsy and treatment naive men.

Materials and Methods: This is a prospective, single institutional study, done from September 2018 to March 2020 in 75 biopsy naive men. The patients with 3, 4 and 5 PIRADS scores underwent mpMRI cognitive target biopsy (mpMRI CTB) followed by standard biopsy (SB) in the same setting by two different urologists. Diagnostic yield of biopsy cores, complications, and stage migration of Gleason's grades was analyzed.

Results: Out of 75 patients, $34.6 \%$ had abnormal digital rectal examination (DRE), and the median serum PSA was 10.6 (4.5-20) ng/mL. Total MRI suspicious lesions were 163. Out of $1263 \mathrm{SB}$ cores, 371 cores were positive for cancer (29.35\%), and out of $326 \mathrm{mpMRI}$ CTB cores, 120 were positive for cancer $(36.8 \%)(\mathrm{P}<0.0001)$. Histopathological examination (HPE) revealed $88 \%, 92 \%$, and $100 \%$ clinically significant cancers in PIRADS 3, 4 and 5 lesions. SB and mpMRI CTB in combination have better cancer detection yield than either of the modality when used alone $(\mathrm{P}-<0.0001)$. Clavien-Dindo grade 1 and grade 4 a complication were seen in $47(62.6 \%)$ and three $(4 \%)$ patients.

Conclusion: In biopsy-naive men with suspected prostate cancer and equivocal DRE, the addition of pre-biopsy mpMRI detects greater numbers of people with clinically significant prostate cancer when compared with SB alone. Combining SB with mpMRI CTB has a superior diagnosing ability when compared with either of the biopsy modalities when used alone.

Keywords: mpMRI target biopsy, prostate cancer, standard systematic biopsy, cognitive targeted biopsy

\section{Introduction}

Prostate cancer is the most frequently occurring male cancer in Europe. ${ }^{1-3}$ TRUS-biopsy can cause significant morbidity, economic impact, and often offers unnecessary biopsies that occasionally miss clinically significant prostate cancers (csPC). ${ }^{4}$ Alternatively, prostate cancers can be screened by Multiparametric MRI (mpMRI) and use the information to localize the cancerous lesion in the gland for a subsequent biopsy, known as an mpMRI targeted biopsy. Most studies state that mpMRI detects high-risk cancers and ignores the low-risk disease. ${ }^{5-9}$ A general misconception is that prostate cancer prevalence is less in developing countries like India. ${ }^{10}$ In addition, mpMRI fusion biopsies are 
not widely available in underdeveloped and developing countries, hence mpMRI Cognitive Target Biopsy (mpMRI CTB) can be used until the fusion technology and medical advancements penetrate the countries with lower economic status. Cognitive target biopsy of prostate is a method where the biopsy performing physician reviews the mpMRI report along with the image, registers the location of the lesion in the prostate in his cognition/mind and then uses the knowledge to track the lesion in TRUS image in order to take target biopsies. The aim of our study is to verify the utility of mpMRI CTB in diagnosing clinically significant prostate cancer in addition to Standard Biopsy (SB) in low and middle income countries.

\section{Materials and Methods}

This is a single-center, prospective, observational study, done between October 2018 and March 2020. The current study was approved by the Institutional Ethics Committee - Clinical studies, Apollo Hospitals, Chennai and our study complied with the Declaration of Helsinki. Written informed consent was obtained from the patients prior to the procedure. All patients who were reviewed in urology department with marginally elevated serum PSA ( $>4 \mathrm{ng} / \mathrm{dl}$ and $<20 \mathrm{ng} / \mathrm{dl}$ ) and with no signs or symptoms of UTI were advised to undergo mpMRI prostate. Among them, all patients with PIRADS score of 3, 4 and 5 were advised to undergo cognitive targeted biopsy (CTB) of prostate based on mpMRI findings.

Inclusion criteria were men of $\geq 18$ years of age referred with clinical suspicion of prostate cancer and who have been advised to have a prostate biopsy with a PIRADS score of 3, 4, and 5, patients with serum PSA $>4$ and $\leq 20 \mathrm{ng} / \mathrm{mL}$, suspected $\leq \mathrm{T} 2$ stage on rectal examination. Exclusion criteria consisted of patients who underwent prior prostate biopsy/surgery or prostate cancer treatments, any contraindications to MRI, coagulopathy, active urinary tract infection (UTI), or patients unfit for the procedure. Patients with elevated serum PSA, normal digital rectal examination (DRE) but with PIRADS 1 and 2, were not subjected to biopsy and were kept on follow up with 6 monthly serum PSA and a yearly physical examination. Whereas patients with abnormal DRE, despite of PIRADS 1 and 2, were biopsied, but excluded from the study. Standardized preoperative testing, procedure technique and post-operative investigations and follow up was done.

The PIRADS (version 2) grading system was followed to report the mpMRI. We used 3T, Philips Ingenia MRI scanner, and the sequence/technique used was high-resolution axial T2W-TSE, T2W-fat-saturated, T1W-TSE, Sagittal T2W-
TSE, coronal - T2W-TSE. Diffusion-weighted imaging, using dynamic contrast enhancement, without the use of an endorectal coil. The mpMRI was reported by a panel of experienced radiologists.

All patients were evaluated with basic blood and urine investigations, renal function test, coagulation profile, and serology. Patients on antiplatelets were evaluated and antiplatelets were stopped for seven days before TRUS biopsy. Antibiotics were given before biopsy (Inj. Cefoperazone Sulbactam $3 / 1.5 \mathrm{gm}$ IV for patients with normal and altered renal parameters). No rectal swabs and no enema or bowel preparation was done. Prostate biopsy was done as an office procedure, in the left lateral decubitus position using a BARD $^{\circledR} \quad$ MAX-CORE ${ }^{\circledR}$ Disposable Core Biopsy Instrument (18Gauge x $25 \mathrm{~cm}$ ), under periprostatic block using $2 \%$ lignocaine injection. Prostate was visualized using a BK ultrasound Biplane 8808e transrectal Ultrasound probe. First, mpMRI CTB was done by a Urologist who took 2 target cores for each suspicious lesion followed by 12 or more Standard Biopsy (SB) cores in the same patient by the second urologist in the same sitting. When the prostate size was more than $50 \mathrm{cc}$, we had taken more than 12 cores. Gleason's score of $\geq 7(4+3)$ was considered as csPC. Oral antibiotics (Cefixime 200mg, BD, for five days) were prescribed along with symptomatic management. Clavien-Dindo classification system was used to classify complications. ${ }^{11}$ Biopsied prostatic cores were graded based on Gleason's score and data analyzed. The patient cohort was followed until they had treatment in the form of either Robotic-assisted laparoscopic radical prostatectomy (RARP) or androgen deprivation therapy or radiotherapy.

\section{Statistical Methods}

Normally and non-normally distributed continuous variables were expressed as Mean \pm Standard deviation and Median (Interquartile range), respectively. Categorical variables were expressed as a percentage. Comparison of categorical variables was done with Chi-square or Fisher's Exact test. MS Excel was used for data entry. Data analysis was done with IBM Statistical Package for the Social Sciences (SPSS, version 25.0, IBM Corp, Armonk, NY, USA). All "p" values $<0.05$ were regarded as statistically significant.

\section{Results}

A total of 75 patients were included in our study. A complete short term analysis was done with none lost to follow up. The mean age was $66.31 \pm 7.9$. Table 1 represents demography data and pre-procedure evaluation. All 75 patients underwent 
Table I Patients Demographic Data

\begin{tabular}{|l|l|}
\hline Parameters & \multicolumn{2}{|c|}{ Numbers/Mean/Percentage } \\
\hline Total Number of patients $(\mathrm{n})$ & 75 \\
Mean age \pm SD (years) & $66.31 \pm 7.9$ \\
Patients with abnormal DRE $(\mathrm{n})$ & $26(34.6 \%)$ \\
Median serum PSA/IQR $(\mathrm{ng} / \mathrm{mL})$ & $10.6(4.5-20)$ \\
Median prostate volume/SD (cc) & $45.67 \pm 18.9$ \\
Total number of MRI suspicious lesions $(\mathrm{n})$ & 163 \\
\hline \multicolumn{2}{|c|}{ Comorbidities $(\mathrm{n})$} \\
\hline Diabetes $(\mathrm{n})$ & 29 \\
Hypertension (n) & 28 \\
Coronary artery disease $(\mathrm{n})$ & 11 \\
Chronic kidney disease $(\mathrm{n})$ & 4 \\
Nil $(\mathrm{n})$ & 27 \\
\hline
\end{tabular}

biopsy as per methodology. In mpMRI CTB, 120 out of 326 cores were found to be positive for prostate cancer (36.8\%). In $\mathrm{SB}, 185$ out of $631 \mathrm{SB}$ cores taken in the left lobe and 186 out of 632 cores taken from the right lobe of the prostate, were positive for malignancy (29.35\%). Tables 2 and 3 shows the core wise and patient wise comparison of SB versus mpMRI CTB $(p=0.0001)$ and $(p=0.0001)$. Patient wise analysis of the cohort revealed that SB alone diagnosed 40 patients with prostate cancer and mpMRI CTB alone diagnosed 34 patients with prostate cancer (Tables 2 and 3 ).

A total of 31 patients were found to be having prostate cancer in both SB and mpMRI CTB. mpMRI CTB diagnosed three more patients than what was diagnosed by SB alone. The computed Kappa value for patient wise analysis was $0.68,95 \%$ CI (0.46-0.79), which showed a substantial agreement between standard biopsy and mpMRI CTB. The concordance rate was $84 \%$. Among the discordant judgment $(16 \%)$, standard biopsy picked nine patients $(75 \%)$ which were not picked by mpMRI CTB and mpMRI CTB picked three $(25 \%)$ patients which were not picked by standard biopsy.

Patients with PIRADS 3, 4 and 5 lesions had 59.5\%, $52.17 \%$ and $70 \%$ prostatic cancer detection rate. The study revealed that, in a higher PIRADS score, a corresponding increase in the diagnosis of csPC (Gleason's score $\geq 7$ ) was seen. The comparatively lower Cancer detection rate (CDR) among PIRADS 4 patients could be due to bias among mpMRI reporting physicians and inter-operator variability.

Table 2 Core Wise Comparison of Standard Biopsy versus Cognitive Targeted Biopsy Using mpMRI Data in Detecting Prostate Cancer

\begin{tabular}{|l|c|c|c|}
\hline Variables & mpMRI CTB & Standard Biopsy & P-value \\
\hline Total cores $(\mathrm{n})$ & 326 & 1263 & $\leq 0.0 \mathrm{I}$ \\
Positive core (n) & 120 & $37 \mathrm{I}$ & \\
Yield in Percentage (\%) & 36.80 & 29.35 & \\
\hline
\end{tabular}

Note: $\mathrm{n}$ - number of cores.

Table 3 Patient Wise Comparison of Standard Biopsy versus Cognitive Targeted Biopsy Using mpMRI Data in Detecting Prostate Cancer

\begin{tabular}{|l|c|c|c|}
\hline Variables & mpMRI CTB +ve & mpMRI CTB -ve & Total (n) \\
\hline Standard biopsy +ve (n) & 31 & 9 & 40 \\
Standard biopsy -ve (n) & 3 & 32 & 35 \\
Total $(n)$ & 34 & 41 & 75 \\
\hline
\end{tabular}

Note: $\mathrm{n}$ - number of patients. 
Table 4 Analysis of PIRADS Score and Detection of Clinically Significant Cancer

\begin{tabular}{|l|c|c|c|c|c|}
\hline $\begin{array}{l}\text { PIRADS } \\
\text { Score } \\
(\mathbf{N}=\mathbf{7 5})\end{array}$ & $\begin{array}{c}\text { No. of Patients } \\
\text { Diagnosed with } \\
\text { Cancer in SB }\end{array}$ & $\begin{array}{c}\text { No. of Patients } \\
\text { Diagnosed with Cancer } \\
\text { in mpMRI CTB }\end{array}$ & $\begin{array}{c}\text { Percentage of } \\
\text { Patients with Cancer } \\
\text { Detection (\%) }\end{array}$ & $\begin{array}{c}\text { No. of Patients } \\
\text { with Gleason's } \\
\text { Score } \geq \mathbf{7}(\mathbf{4 + 3})\end{array}$ & $\begin{array}{c}\text { No. of Patients } \\
\text { with Gleason's } \\
\text { Score <7 }\end{array}$ \\
\hline $3(n=42)$ & 23 & 17 & $59.52(n=25)$ & $22 / 25(88 \%)$ & $3 / 25(12 \%)$ \\
$4(n=23)$ & 11 & 12 & $52.17(n=13)$ & $12 / 13(92.30 \%)$ & $1 / 23(7.70 \%)$ \\
$5(n=10)$ & 6 & 5 & $70(n=7)$ & $7 / 7(100 \%)$ & $0 / 7(0 \%)$ \\
\hline
\end{tabular}

(Table 4, Table 5, Table 6 shows the analysis of the PIRADS score and the detection of csPC. Combining mpMRI CTB along with SB had a better CDR when compared with either of the modalities alone (p-value 0.0001) (Tables 5 and 6). The computed Kappa value for SB vs combined biopsy was 0.92 (95\% CI 0.75-0.93) which showed almost perfect agreement between SB alone and combined targeted biopsy. The concordance rate by both procedures was $96 \%$ and discordance was $4 \%$. Three cases which were missed by SB alone were diagnosed by SB and CTB (combined biopsy). Similarly, the computed Kappa value for mpMRI CTB vs combined biopsy was $0.76,95 \%$ CI $(0.58-0.76)$ which showed substantial agreement between mpMRI CTB and combined biopsy. Here, nine cases which were missed by mpMRI CTB alone was diagnosed while combining SB and CTB (combined biopsy).
The Clavien-Dindo Classification was used to grade complications. Twenty-five patients had no complications. About 41 patients $(55 \%)$ had self-limiting transient hematuria, 24 patients (32\%) had rectal bleeding, seven patients (5.3\%) had urinary retention warranting catheter deployment and three patients (4\%) had urosepsis warranting admission with uneventful recovery. These patients were given IV antibiotics, hydration and discharged in stable condition within 24 hrs. Fifty patients had Clavien-Dindo grade 1 complication and three patients had Grade 4a complication. Among 46 patients who were diagnosed with prostate cancer, 25 patients (54.3\%) underwent RARP, 17 patients opted for androgen deprivation therapy and four patients opted for active surveillance. The post RARP histopathology report (HPR) showed a 12\% up-gradation and 9.3\% down-gradation of the Gleason's score.

Table 5 Patient Wise Comparisons of SB versus Combined Biopsy (Standard + CTB) Using mpMRI Data in Detecting Prostate Cancer

\begin{tabular}{|l|c|c|c|}
\hline Variables & Combined Biopsy +ve & Combined Biopsy -ve & Total \\
\hline Standard biopsy +ve (n) & 40 & 0 & 40 \\
Standard biopsy -ve (n) & 3 & 32 & 35 \\
Total $(n)$ & 43 & 32 & 75 \\
\hline P-value & \multicolumn{3}{|c|}{$\leq 0001^{\#}$} \\
\hline
\end{tabular}

Notes: $\mathrm{n}$ - number of patients. ${ }^{\#}$ Combined biopsy had significantly higher cancer detection rate. Kappa statistics $=0.92$ (agreement between two methods is $92 \%$ ), $95 \% \mathrm{Cl}$ $(0.75-0.93)$.

Abbreviation: $\mathrm{Cl}$, confidence interval.

Table 6 Patient Wise Comparisons of mpMRI CTB versus Combined Biopsy (Standard + CTB) in Detecting Prostate Cancer

\begin{tabular}{|l|c|c|c|}
\hline Variables & Combined Biopsy +ve & Combined Biopsy -ve & Total \\
\hline mpMRI CTB +ve(n) & 34 & 0 & 34 \\
mpMRI CTB -ve (n) & 9 & 32 & 41 \\
Total (n) & 43 & 32 & 75 \\
\hline P-value & \multicolumn{3}{|c|}{$\leq 0.000 \mathrm{I}^{\#}$} \\
\hline
\end{tabular}

Notes: $\mathrm{n}$ - number of patients. ${ }^{\#}$ Combined biopsy had significantly higher cancer detection rate. Kappa statistics $=0.76$ (agreement between two methods is $76 \%$ ), $95 \% \mathrm{Cl}$ (0.58-0.76).

Abbreviation: $\mathrm{Cl}$, confidence interval. 


\section{Discussion}

mpMRI has been a very promising diagnostic tool for prostate cancer, allowing clinicians to direct biopsies towards suspected lesions rather than operating randomly. There has been considerable concern regarding overdiagnosis and subsequent overtreatment of men with clinically indolent prostate cancer; hence, better characterization of prostate cancer is highly desirable. ${ }^{12} \mathrm{MRI} / \mathrm{US}$-fusion-guided, targeted biopsies may potentially offer such an improvement. ${ }^{5}$

This study was conducted in South India, with a diverse population coming from many SAARC nations and NorthEastern India. Baring a few, most studies on mpMRI and TRUS prostate biopsies are based on western literature. mpMRI fusion biopsy is limited in India due to high-cost factors. We have evaluated the diagnostic yield of CTB using mpMRI data while comparing it to Standard biopsy data, which can be done, if a TRUS machine is available, with a trained Urologist who knows to read mpMRI.

The median serum PSA in our study was $10.6 \mathrm{ng} / \mathrm{mL}$ (IQR 4.5-20), which was slightly higher compared to other studies. Mean prostate volume in our study was $45.67 \pm 18.9$ cc and $34.6 \%$ had an abnormal DRE with a total of 163 mpMRI suspicious lesions. The overall CDR in our study, (yield from both SB and mpMRI CTB) was $61.33 \%(n=46)$. Twenty-nine patients showed no malignancy. The overall $\mathrm{CDR}$ of $\operatorname{csPC}$ was $56 \%(\mathrm{n}=42)$, and when considering $\mathrm{SB}$ alone, was $53.33 \%(\mathrm{n}=40)$, and considering mpMRI CTB alone was $45.33 \%$ ( $\mathrm{n}=34)$ ( $\mathrm{p}$-value $<0.0001)$. Similarly, csPC was detected in $38 \%$ and $26 \%$ of patients who underwent mpMRI target biopsy and SB respectively, in The PRECISION study by Kasivisvanathan et al. ${ }^{13}$

Tonttila et al found that the overall and clinically significant CDRs in SB were 57\%, and $45 \%$ respectively, while for TB it was $64 \%$ and $55 \%$ respectively, which was not clinically significant (p-value of 0.8$).{ }^{7}$ Van der Leest et al study also concluded that mpMRI guided TB when compared with SB yielded similar clinically significant prostate CDR. ${ }^{14}$
But the studies by Bansal et al, Siddiqui et al, Porpiglia et al and Ahmed et al found that CDR improved by the addition of mpMRI guided TB, to the SB. Bansal et al, and Porpiglia et al study showed a clinically significant CDR with SB alone at $60.2 \%$ and $20 \%$ respectively, while with TB alone, it was $71.2 \%$ and $46 \%$ respectively (p-value 0.033 and $p$-value $<0.001$ ) (Table 7) ${ }^{6,15}$ Similarly, Ahmed et al study showed CDR with SB and TB alone at $9.2 \%$ and $47.5 \%$ respectively. ${ }^{5}$

The analysis of the cores in our study revealed that SB vs mpMRI CTB was having an overall CDR of $29.35 \%$ vs $36.80 \%$ (p-value of 0.01 ) (Tables 2 and 3). Tonttila et al study showed a SB vs mpMRI CTB cancer detection rate of $57 \%$ vs $51 \%$ (p-value of 0.9 ). ${ }^{7}$ Bansal et al, and Porpiglia et al study showed that TB had a significantly higher cancer yield when compared with SB (Bansal et al SB vs TB $-16.2 \%$ vs $44.3 \%$ ).

Roethke et al concluded that the PIRADS score is a useful prognostic tool for prostate cancer risk stratification, and it improved the detection of csPC. ${ }^{16}$ PIRADS score-wise analysis of cores and clinically significant CDRs of our study was comparable with Bansal et al. The yield of csPC for PIRADS 3, 4 and 5 was $88 \%, 92.3 \%$, and $100 \%$ in our study and $58 \%, 75 \%$ and $88 \%$ in Bansal et al study. Porpiglia et al study also revealed $12.5 \%, 75 \%$, and $81.3 \%$ clinically significant CDR for PIRADS 3, 4 and 5.

MRI-FIRST trial done by Rouvière et al found that SB detected fewer clinically significant cancers when compared with mpMRI TB $(15.2 \%$ VS $21 \% ; p=0.0036)$ and SB detected more patients with clinically non-significant cancers when compared with mpMRI TB (20\% vs 5.8\%; $\mathrm{p}<0.0001){ }^{17}$

Limiting prostate biopsy to only mpMRI CTB protocol misses some csPC. It may be due to the presence of several significant cancers outside MRI suspicious lesions. ${ }^{18}$ In our study, nine patients were detected only by SB and were missed by mpMRI CTB, of which 6 patients had csPC. Apical and anterior prostate cancers are often missed by

Table 7 Comparison of Demographic Data Among Other Studies

\begin{tabular}{|c|c|c|c|c|c|}
\hline Variables & Our Study & $\begin{array}{c}\text { Tonttila, Panu } \\
\text { P, et } \text { al }^{7}\end{array}$ & $\begin{array}{c}\text { Bansal, Somendra, } \\
\text { et al }^{15}\end{array}$ & $\begin{array}{c}\text { Siddiqui, M. Minhaj, } \\
\text { et al }^{9}\end{array}$ & $\begin{array}{c}\text { Porpiglia, Francesco, } \\
\text { et } \mathrm{al}^{6}\end{array}$ \\
\hline Sample size (n) & 75 & 113 & 96 & 582 & 223 \\
\hline Age \pm SD (Years)/Range & $66.31 \pm 7.9$ & $63 \pm 6.8$ & $64.4 \pm 7.5$ & $61.3 \pm 8.4$ & $60(58-70)$ \\
\hline Median Serum P.S.A with IQR(ng/mL) & $10.6(4.5-20)$ & $6.5(4.2-10.7)$ & $8.6(6.3-12.5)$ & 9.9 (mean) & $6.3(4.8-8.5)$ \\
\hline Mean Prostate vol \pm SD/mean with IQR(cc) & $45.67 \pm 18.9 \mathrm{cc}$ & $29.8(23.5-44.3)$ & $41(30.4-55)$ & $56.4 \pm 31.2$ & $46.2(34.5-71.6)$ \\
\hline Abnormal DRE \% & 34.6 & 12 & 24 & $\mathrm{Na}$ & $\mathrm{Na}$ \\
\hline Suspicious MRI lesions(n) & 163 & 53 & 176 & $\mathrm{Na}$ & $\mathrm{Na}$ \\
\hline
\end{tabular}


conventional TRUS biopsy. ${ }^{19}$ MRI-TRUS fusion biopsy is most useful in targeting suspicious lesions of the anterior prostate and is ideally suited for patients with a history of previous negative TRUS biopsy and persistently elevated PSA. $^{20}$ Van der Leest et al in their study proved that SB yields of csPC in PIRADS 1 and 2 patients are low (4\%) and hence biopsy can be avoided, thus reducing complicated UTIs and sepsis in around $2.9 \%$ of patients. This study also found that "Focal saturation" improved detection of csPC while doing an mpMRI-TB. ${ }^{14}$

In contemporary series, the frequency of urosepsis varied between $0.2 \%$ and $3.06 \%$. The lowest rates of urosepsis reported were by Zaytoun et al, and Raaijmakers et al $(0.2 \%$ and $0.5 \%$ respectively $){ }^{21,22}$ Bansal et al study had one case of urosepsis (1.04\%). Ahmed et al study and Van der Leest et al study had complication rates of $5.9 \%$ and $6 \%$ respectively. ${ }^{14}$ Three patients developed urosepsis in our study (4\%). Rates of sepsis among different studies varied, which may be due to different biopsy techniques, consistent reporting, the definition of urosepsis used, and the prophylactic regimes used to prevent infections. ${ }^{23}$

A $12 \%$ up-gradation and a $9.3 \%$ down-gradation of the Gleason's score was noticed in our study, which differed from Khoddami et al $(9.1 \%$ over-graded, and $22.7 \%$ under graded). ${ }^{24}$ This may be due to sampling error, due to the heterogeneous character of prostatic adenocarcinoma. Divrik et al showed improvement in the agreement between the Gleason score from core biopsy and radical prostatectomy specimens with an increase in the number of core biopsies obtained (56\% vs $41 \%) .{ }^{25}$ To minimize grading error, taking at least 6 cores each $1.5 \mathrm{~cm}$ in length is recommended.

The limitations of this study are that it is a single institute study with a small cohort, the involvement of more than one urologist and radiologist, and lack of PIRADS 1 and 2 in the biopsied cohorts. Clinically significant cancers could have been missed by both mpMRI CTB and SB since Template prostate mapping biopsy was not done. Only $54 \%$ of patients diagnosed with carcinoma prostate by TRUS biopsy went on to have RARP.

\section{Conclusion}

In biopsy and treatment naive men with suspected prostate cancer, Serum PSA levels $<20 \mathrm{ng} / \mathrm{mL}$, and equivocal DRE results, the addition of pre-biopsy mpMRI allowed us to detect greater numbers of people with csPC when compared with SB alone. Combining SB with mpMRI CTB data, gave a superior diagnosing ability when compared with either of the biopsy modality when used alone. There are not many studies from the low and middle income countries that analyze the impact of cognitively targeted biopsies using mpMRI data on the detection of clinically significant cancer while comparing against Standard biopsies, hence this analysis will help in initiating further studies and pave way for incorporating mpMRI CTB, till mpMRI fusion biopsy is more widely available.

\section{Abbreviations}

DRE, digital rectal examination; PSA, prostate-specific antigen; TRUS, transrectal ultrasound; CDR, cancer detection rate; csPC, clinically significant prostate cancer; mpMRI, multiparametric magnetic resonance imaging; mpMRI CTB, mpMRI cognitive target biopsy; CTB, cognitive target biopsy; SB, standard biopsy; TB, target biopsy; RARP, robotic-assisted laparoscopic radical prostatectomy; PIRADS, Prostate Imaging Reporting and Data System; HP, histopathology report.

\section{Data Sharing Statement}

The datasets used and/or analyzed during the current study are available from the corresponding author. The datasets intended for sharing are deidentified.

\section{Ethical Committee Approval}

Ethics approval and consent to participate in the study: written consent obtained. Name of the committee which gave ethical clearance for study: "Institutional Ethics Committee - Clinical studies". The procedures and ethical clearance were in accordance with the Helsinki declaration.

\section{Consent for Publication}

Written consent was obtained from the participants of the study.

\section{Author Contributions}

All authors made substantial contributions to conception and design, acquisition of data, or analysis and interpretation of data; took part in drafting the article or revising it critically for important intellectual content; agreed to submit to the current journal; gave final approval of the version to be published; and agree to be accountable for all aspects of the work.

\section{Funding}

There is no funding to report.

\section{Disclosure}

The authors report no conflicts of interest in this work. 


\section{References}

1. Center MM, Jemal A, Lortet-Tieulent J, et al. International variation in prostate cancer incidence and mortality rates. Eur Urol. 2012;61 (6):1079-1092. doi:10.1016/j.eururo.2012.02.054

2. Villers A, Grosclaude P. Epidemiology of prostate cancer: review article. Nuclear medicine. 2008 Jan 1; 32 (1): 2-4.

3. Carter HB, Hamper UM, Sheth S, Sanders RC, Epstein JI, Walsh PC. Evaluation of transrectal ultrasound in the early detection of prostate cancer. J Urol. 1989;142(4):1008-1010. doi:10.1016/S0022-5347[17] 38971-1

4. Schoots IG, Roobol MJ, Nieboer D, Bangma CH, Steyerberg EW, Hunink MGM. Magnetic resonance imaging-targeted biopsy may enhance the diagnostic accuracy of significant prostate cancer detection compared to standard transrectal ultrasound-guided biopsy: a systematic review and meta-analysis. Eur Urol. 2015;68(3):438450. doi:10.1016/j.eururo.2014.11.037

5. Ahmed HU, El-Shater Bosaily A, Brown LC, et al. Diagnostic accuracy of multi-parametric MRI and TRUS biopsy in prostate cancer [PROMIS]: a paired validating confirmatory study. Lancet. 2017;389(10071):815-822. doi:10.1016/S0140-6736[16]32401-1

6. Porpiglia F, Manfredi M, Mele F, et al. Diagnostic pathway with multiparametric magnetic resonance imaging versus standard pathway: results from a Randomized Prospective Study in Biopsy-naïve Patients with Suspected Prostate Cancer. Eur Urol. 2017;72(2):282288. doi:10.1016/j.eururo.2016.08.041

7. Tonttila PP, Lantto J, Pääkkö E, et al. Prebiopsy multiparametric magnetic resonance imaging for prostate cancer diagnosis in biopsy-naive men with suspected prostate cancer based on elevated prostate-specific antigen values: results from a Randomized Prospective Blinded Controlled Trial. Eur Urol. 2016;69(3):419425. doi:10.1016/j.eururo.2015.05.024

8. Boesen L, Noergaard N, Chabanova E, et al. Early experience with multiparametric magnetic resonance imaging-targeted biopsies under visual transrectal ultrasound guidance in patients suspicious for prostate cancer undergoing repeated biopsy. Scand J Urol. 2015;49 (1):25-34. doi:10.3109/21681805.2014.925497

9. Siddiqui MM, Rais-Bahrami S, Truong H, et al. Magnetic resonance imaging/ultrasound-fusion biopsy significantly upgrades prostate cancer versus systematic 12-core transrectal ultrasound biopsy. Eur Urol. 2013;64(5):713-719. doi:10.1016/j.eururo.2013.05.059

10. Jain S, Saxena S, Kumar A. Epidemiology of prostate cancer in India. Meta Gene. 2014;2:596-605. doi:10.1016/j.mgene.2014.07.007

11. Dindo D, Demartines N, Clavien PA. Classification of surgical complications: a new proposal with evaluation in a cohort of 6336 patients and results of a survey. Ann Surg. 2004;240(2):205-213. doi:10.1097/01.sla.0000133083.54934.ae

12. Siegel RL, Miller KD, Jemal A. Cancer statistics, 2019. CA Cancer J Clin. 2019;69(1):7-34. doi:10.3322/caac.21551

13. Kasivisvanathan V, Rannikko AS, Borghi M, et al. MRI-targeted or standard biopsy for prostate-cancer diagnosis. $N$ Engl $\mathrm{J} \mathrm{Med}$. 2018;378(19):1767-1777. doi:10.1056/NEJMoa1801993
14. van der Leest M, Cornel E, Israël B, et al. Head-to-head comparison of transrectal ultrasound-guided prostate biopsy versus multiparametric prostate resonance imaging with subsequent magnetic resonance-guided biopsy in biopsy-naïve men with elevated prostatespecific antigen: a large prospective Multicenter Clinical Study. Eur Urol. 2019;75(4):570-578. doi:10.1016/j.eururo.2018.11.023

15. Bansal S, Gupta NP, Yadav R, et al. Multiparametric magnetic resonance imaging-transrectal ultrasound fusion prostate biopsy: a prospective, single centre study. Indian J Urol. 2017;33(2):134-139. doi:10.4103/0970-1591.203414

16. Roethke MC, Kuru TH, Schultze S, et al. Evaluation of the ESUR PIRADS scoring system for multiparametric MRI of the prostate with targeted MR/TRUS fusion-guided biopsy at 3.0 Tesla. Eur Radiol. 2014;24(2):344-352. doi:10.1007/s00330-013-3017-5

17. Rouvière $\mathrm{O}$, Puech $\mathrm{P}$, Renard-Penna $\mathrm{R}$, et al. Use of prostate systematic and targeted biopsy on the basis of multiparametric MRI in biopsy-naive patients [MRI-FIRST]: a prospective, multicentre, paired diagnostic study. Lancet Oncol. 2019;20(1):100-109. doi:10.1016/S1470-2045(18)30569-2

18. Sonn GA, Natarajan S, Margolis DJA, et al. Targeted biopsy in the detection of prostate cancer using an office based magnetic resonance ultrasound fusion device. $J$ Urol. 2013;189(1):86-92. doi:10.1016/j. juro.2012.08.095

19. Yacoub JH, Verma S, Moulton JS, Eggener S, Oto A. Imaging-guided prostate biopsy: conventional and Emerging Techniques. Radiographics. 2012;32(3):819-837. doi:10.1148/rg.323115053

20. Marks L, Young S, Natarajan S. MRI-ultrasound fusion for guidance of targeted prostate biopsy. Curr Opin Urol. 2013;23(1):43-50. doi:10.1097/MOU.0b013e32835ad3ee

21. Zaytoun OM, Vargo EH, Rajan R, Berglund R, Gordon S, Jones JS. Emergence of fluoroquinolone-resistant Escherichia coli as cause of postprostate biopsy infection: implications for prophylaxis and treatment. Urology. 2011 May 1;77(5):1035-41.

22. Raaijmakers R, Kirkels WJ, Roobol MJ, Wildhagen MF, Schrder FH. Complication rates and risk factors of 5802 transrectal ultrasoundguided sextant biopsies of the prostate within a population-based screening program. Urology. 2002 Nov 1;60(5):826-30.

23. Carmignani L, Picozzi S, Spinelli M, et al. Bacterial sepsis following prostatic biopsy. Int Urol Nephrol. 2012;44(4):1055-1063. doi:10.1007/s11255-012-0145-9

24. Khoddami M, Khademi Y, Aghdam MK, et al. Correlation between Gleason scores in needle biopsy and corresponding radical prostatectomy specimens: a twelve-year review. Iran J Pathol. 2016;11 (2): 120 .

25. Divrik RT, Erog lu A, Şahin A, Zorlu F, Özen H. Increasing the number of biopsies increases the concordance of Gleason scores of needle biopsies and prostatectomy specimens. InUrologic Oncology: Seminars and Original Investigations 2007 Sep 1 (Vol. 25, No. 5, pp. 376-382). Elsevier.
Research and Reports in Urology

\section{Publish your work in this journal}

Research and Reports in Urology is an international, peer-reviewed, open access journal publishing original research, reports, editorials, reviews and commentaries on all aspects of adult and pediatric urology in the clinic and laboratory including the following topics: Pathology, pathophysiology of urological disease; Investigation and treatment of urological disease; Pharmacology of drugs used for the treatment of urological disease. The manuscript management system is completely online and includes a very quick and fair peer-review system, which is all easy to use. Visit http://www.dovepress.com/ testimonials.php to read real quotes from published authors. 